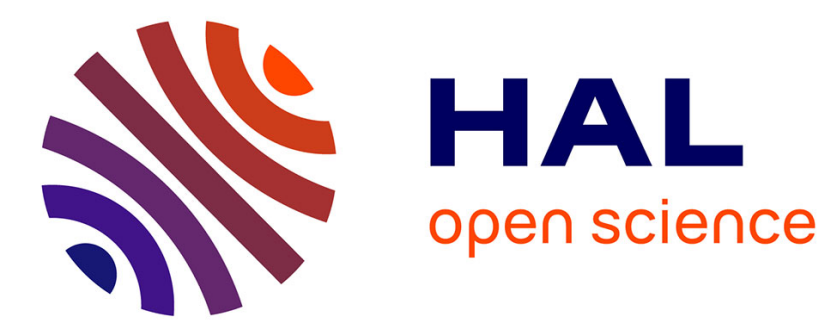

\title{
Tomographic reconstruction using a generalized regressive discrete Fourier series
}

Joris Vanherzeele, Roberto Longo, Steve S.V Vanlanduit, Patrick Guillaume

\section{To cite this version:}

Joris Vanherzeele, Roberto Longo, Steve S.V Vanlanduit, Patrick Guillaume. Tomographic reconstruction using a generalized regressive discrete Fourier series. Mechanical Systems and Signal Processing, 2008, 22 (Issue 5 ), pp.1237-1247. 10.1016/j.ymssp.2007.11.002 . hal-01586222

\section{HAL Id: hal-01586222 \\ https://hal.science/hal-01586222}

Submitted on 10 Feb 2018

HAL is a multi-disciplinary open access archive for the deposit and dissemination of scientific research documents, whether they are published or not. The documents may come from teaching and research institutions in France or abroad, or from public or private research centers.
L'archive ouverte pluridisciplinaire HAL, est destinée au dépôt et à la diffusion de documents scientifiques de niveau recherche, publiés ou non, émanant des établissements d'enseignement et de recherche français ou étrangers, des laboratoires publics ou privés. 


\title{
Tomographic reconstruction using a generalized regressive discrete Fourier series
}

\author{
J. Vanherzeele*, R. Longo, S. Vanlanduit, P. Guillaume \\ Department of Mechanical Engineering, Vrije Universiteit Brussel, Pleinlaan 2, 1050 Brussel, Belgium
}

\begin{abstract}
When measuring three-dimensional phenomena such as flow fields or acoustic fields using an interferometric technique, one is proneto measure different angles of view to obtain a full three-dimensional representation of the phenomenon under investigation. This is due to the fact that an interferometric technique measures a line integral across the optical path. To obtain the full three-dimensional view the different angles of view are passed through a tomographic algorithm. The most widely used tomographic method is the so-called filtered back projection. However, this process suffers from a series of drawbacks, the most important one being the fact that substantial truncation errors occur in the back projection step. In this article a method is devised to eliminate these errors, based on a parametric frequency-domain approach called generalized regressive discrete Fourier series (GRDFS). The method will be applied both to simulated acoustic fields as to real acoustic fields measured using laser doppler vibrometry. The acoustic source will be rotated to obtain continuous angle views of the acoustic field, hence eliminating the tedious process of rotating the measurement setup.
\end{abstract}

Keywords: Scanning laser doppler vibrometer; Rotating acoustic source identification;

Tomographic reconstruction; Generalized regressive discrete Fourier series

\section{Introduction}

When measuring density signals with interferometric techniques line integrals over the laser beam optical path are acquired. Therefore images are often taken at different angles to derive local density distribution, which in turn implies the need for tomographic reconstruction algorithms [1]. The tomographic algorithm which is used most often is called the filtered back projection method. However, this approach suffers from a major drawback in casu the fact that truncation/interpolation errors appear when projecting the data back from the measured polar coordinates to cartesian coordinates. In this article a parametric frequency-domain method will be incorporated in the tomography process to eliminate these errors. This frequency-domain method, called a regressive discrete Fourier series (RDFS), was first introduced by Arruda [2,3] and bears the advantage of producing leakage-reduced representation of certain sequences. The RDFS was later elaborated

\footnotetext{
*Corresponding author. Tel.: + 32 26292807; fax: + 3226292865

E-mail address: Joris.Vanherzeele@vub.ac.be (J. Vanherzeele).
} 
to a generalized version (GRDFS) in which the data in question were represented by a series of complex exponentials whose frequency, damping and amplitude were to be determined. It was shown that this series could be transformed into a pole-residue model, hence obtaining a completely leakage-free estimate of the data [4]. Using the latter GRDFS it will be proven possible in this article to obtain a far more accurate image of the phenomenon under inspection. The accuracy of the method will be validated in both simulations and experiments. In this article, measurements, using a scanning LDV, on an acoustic field during rotation of the source are shown. In this way a continuous and automatic measurement sequence of the angular views necessary for the three-dimensional reconstruction is obtained. The measurement principle $[5,6]$ together with the measurement set-up of the rotating acoustic source will be repeated shortly [7]. In the next section the classical filtered back projection tomography principle and algorithm will be explained. Thereafter the use of the GRDFS will be shown.

\section{Tomography}

Consider the parallel-beam geometry in Fig. 1. The function $f(x, y)$ represents the field function under inspection.

The function $p(r, \theta)$ is called the projection of the function $f(x, y)$ for a given angle $\theta$. Usually the projection profile is determined for angles in a range $[0 \ldots \pi]$, in order to obtain full three-dimensional information. Stacking all the projections one by one results in a two-dimensional data set which is called the Radontransform of the function $f(x, y): R(f(x, y))$. It can be described by the following equation, which simply denotes the coordinate transform:

$$
p(r, \theta)=R(f(x, y))=\sum_{s} f(r \cos \theta-s \sin \theta, r \sin \theta+s \cos \theta) \Delta s .
$$

In this equation the function $f(x, y)$ is integrated along the $s$-direction and projected onto the $r$-axis. The projections are at an angle $\theta$ with respect to the original axes and the spatial resolution is denoted by $\Delta r$.

\subsection{Filtered back projection}

Using this method the measurements obtained at each scanned point are projected back along the same line (or equivalently the same $\theta$ ). This implies that the measured values are 'smeared' across the unknown density line.

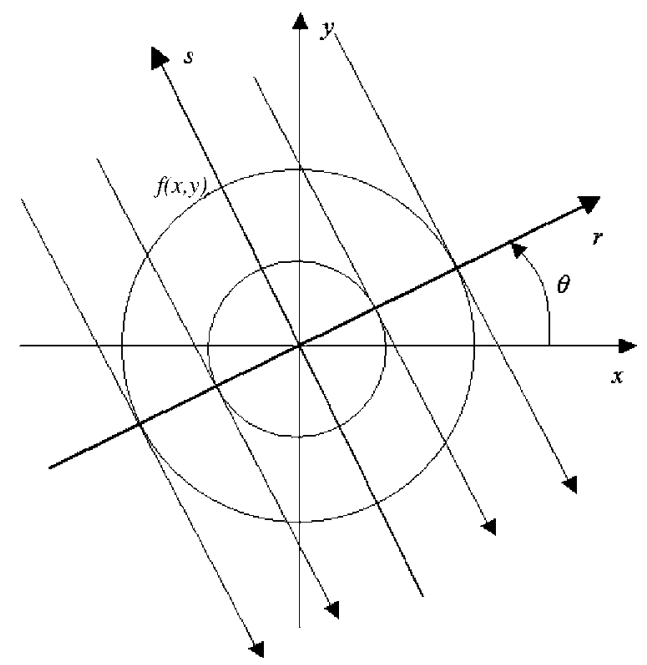

Fig. 1. Projection coordinate system. 
Mathematically the back projection of a single measured projection along an unknown density is given by:

$$
b(x, y, \theta)=\int_{-\infty}^{\infty} p(r, \theta) \delta(x \cos \theta+y \sin \theta-r) d r,
$$

where $b(x, y, \theta)$ is the back-projected density due to the projection $p(r, \theta)$ and $\delta$ indicates the Dirac delta function. Essentially this statement implies that the point of density is somewhere along the line, due to this an assignment of the measured value along the entire line results in a 'crude' reconstruction.

By adding these densities across all angles, a laminogram is obtained:

$$
\begin{aligned}
f_{b}(x, y) & =\int_{0}^{\pi} b(x, y, \theta) d \theta \\
& =\int_{0}^{\pi} \int_{-\infty}^{\infty} p(r, \theta) \delta(x \cos \theta+y \sin \theta-r) d r d \theta .
\end{aligned}
$$

This function $f_{b}(x, y)$ is a distorted image of the field function $f(x, y)$. The relationship between both can be determined by first formulating the back projection of the dirac function itself:

$$
\begin{aligned}
h_{b}(x, y) & =\int_{0}^{\pi} \int_{-\infty}^{\infty} \delta(r) \delta(x \cos \theta+y \sin \theta-r) d r d \theta \\
& =\frac{1}{r} .
\end{aligned}
$$

Therefore the distorted image $f_{b}(x, y)$ can be written as

$$
f_{b}(x, y)=f(x, y) * \frac{1}{r},
$$

where $*$ indicates a convolution product.

Eq. (5) shows that the calculated image is a blurred version of the original. This blurring can be remedied by applying a filtered back projection algorithm. Starting from Eq. (3), the projection data can be substituted using the so-called central slice theorem (CST) algorithm. The CST theorem states that the value of the twodimensional Fourier transform of $f(x, y)$ along a line at an inclination angle $\theta$ is given by the one-dimensional Fourier transform of $p(r, \theta)$. This results in

$$
f_{b}(x, y)=\int_{0}^{\pi} \int_{-\infty}^{\infty}\left[\int_{-\infty}^{\infty} P(k, \theta) e^{i 2 \pi k r} d k\right] \delta(x \cos \theta+y \sin \theta-r) d r d \theta .
$$

When the integration over $r$ is performed, the following relation is obtained:

$$
f_{b}(x, y)=\int_{0}^{\pi} \int_{-\infty}^{\infty} P(k, \theta) e^{i 2 \pi k(x \cos \theta+y \sin \theta)} d \theta d k .
$$

Eq. (7) can be compared to the polar representation of the 2D Fourier transform of the field function $f(x, y)$ in which the Fourier coefficients have been substituted by the projection data via the CST theorem:

$$
f(x, y)=\int_{0}^{\pi} \int_{-\infty}^{\infty}|k| P(k, \theta) e^{i 2 \pi k(x \cos \theta+y \sin \theta)} d \theta d k .
$$

Both equations differ only by the $|k|$ factor as could be expected. The blurring is fixed by weighting the Fourier transform of the projection data by a filter $|k|$ prior to inverse Fourier transforming the sequence and back projecting.

When looking at Eq. (8) it can be denoted that once the projected data have been filtered by the ramp filter $|k|$ a second step is needed to transform this filtered projected data back to cartesian coordinates (denoted by the exponential in Eq. (8)).

Since the actual measurements are discrete, the obtained data will be discrete. Therefore, the latter transformation inherently implies obtaining discrete coordinates which will unfortunately be non-integer. These non-integer coordinates are then truncated or interpolated linearly or with splines, in order to be able to use them in the filtered projected data. This step introduces some non-negligible errors. This can, however, be 
solved by representing $|k| P(k, \theta)$ by a parametric model, hence making it possible to determine the value of the filtered projected data at the non-integer positions. This can be done by using a generalized regressive discrete Fourier series approach [4]. In the next section this approach will be explained and applied to the filtered back projection method.

\section{Back projection using a generalized regressive discrete Fourier series}

In this section, a parametric frequency-domain representation, starting from the discrete Fourier coefficients $|k| P(k, \theta)$ of the filtered projected data is shown. The method boils down to the following steps:

- Take the filtered projection data $|k| P(k, \theta)$ and represent its inverse Fourier transform at an angle $\theta$ by a series of complex exponential functions with unknown frequency $\omega$, damping $\sigma$ and amplitude $a$.

- Take the discrete Fourier transform (DFT) of this sequence.

- Transform into a pole-residue model and estimate poles and residues using a least squares approach (or maximum likelihood (ML)).

- Back project the parametric synthesized sequence using the non-integer coordinates $r=x \cos \theta+y \sin \theta$.

The inverse Fourier transform of the filtered projected data $|k| P(k, \theta)$ for a certain angle $\theta$ can be written as

$$
\sum_{k}|k| P(k, \theta) e^{i 2 \pi k r / N}=a e^{-\sigma r \Delta r+i \omega r \Delta r}=a \lambda^{r}
$$

with $r=0, \ldots, N-1, N$ denoting the number of grid points in the $r$-direction and $\lambda=e^{i \omega \Delta r}, \Delta r$ denoting the sample period of the data. By taking a DFT of the acquired signal, a transfer function model is derived (Eq. (10)). From this model the poles and residues (or amplitudes) are extracted. The parameter $\theta$ will be left out in the following equations as it has no contribution to the derivation:

$$
|k| P(k)=\sum_{r=0}^{N-1} a \lambda^{r} z_{k}^{-r},
$$

where $z_{k}=e^{i 2 \pi k / N}$. This expression (Eq. (10)) can be written as follows by expanding the sum:

$$
|k| P(k)=a \frac{1-\lambda^{N}}{1-\lambda z_{k}^{-1}} .
$$

Now, in the more general situation, the filtered projected data sequence will contain more than one frequency component. Therefore the previous equation can be rewritten as follows for multiple components $l$ :

$$
|k| P(k)=\sum_{l=1}^{L} \frac{\overline{a_{l}}}{1-\lambda_{l} z_{k}^{-1}}
$$

with $\overline{a_{l}}=a_{l}\left(1-\lambda_{l}^{N}\right)$.

Eq. (12) is nothing other than the pole/residue representation of the DFT $|k| P(k)$. Therefore it is possible by means of a simple least squares approach (or for more reliable results a ML approach [8,9]) to retrieve values for the poles $\lambda_{l}$ and the residues $\overline{a_{l}}$. The true amplitudes can be obtained by $a_{l}=\overline{a_{l}} /\left(1-\lambda_{l}^{N}\right)$. By inserting these estimates back in Eq. (9) a parametric representation of the filtered projected data is obtained. The latter equation clearly allows non-integer $r$-coordinates $(r=x \cos \theta+y \sin \theta)$ to be used. This shows that using the GRDFS the true calculated coordinates can be introduced and hence any truncation or interpolation errors are eliminated. In the next section simulations will be shown, on damped sinusoids using both the classical technique as well as the proposed approach. 


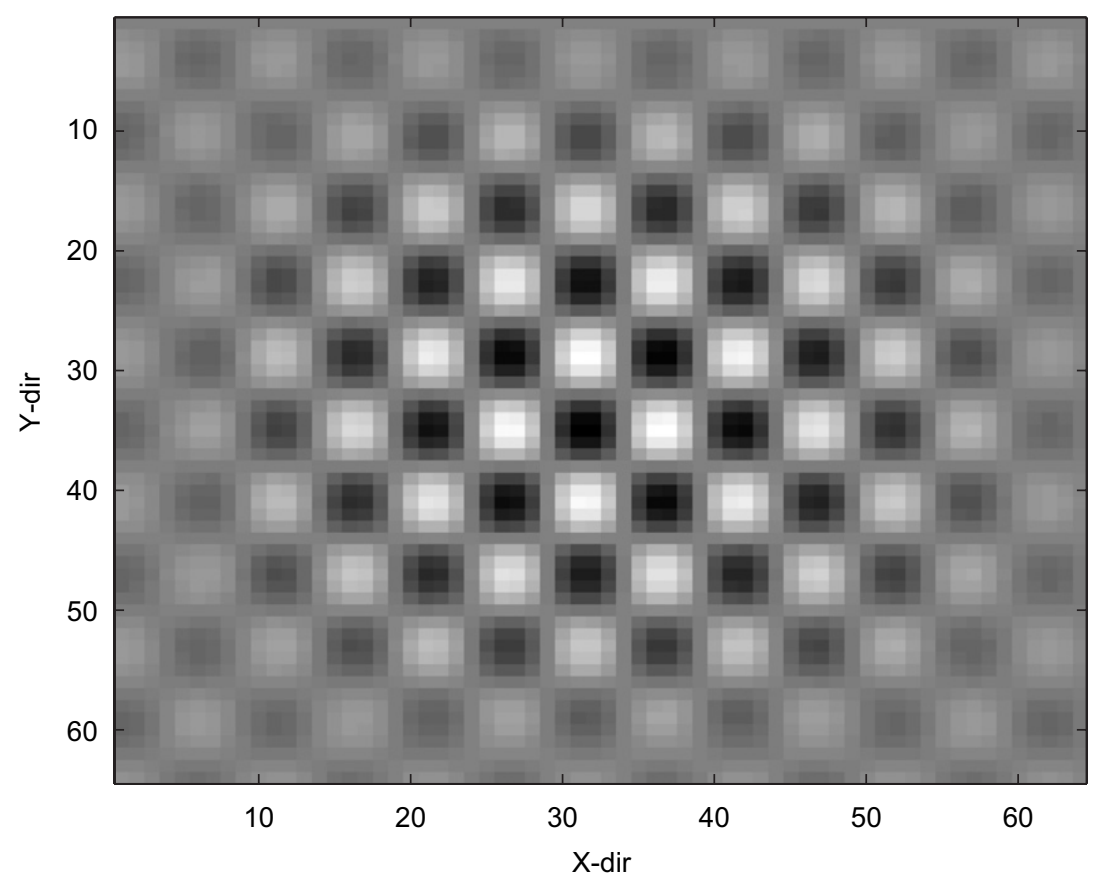

Fig. 2. Damped sinusoidal field.

\section{Simulations}

In this section simulations will be shown on a damped sinusoidal field. The image can be see in Fig. 2. This figure is composed of two sinusoids with 6.3 periods in the $X$-direction and 5.2 periods in the $Y$-direction, with $20 \%$ damping.

The projection data were obtained using the radon function available in the matlab image processing toolbox. First, an angular resolution of $10^{\circ}$ was chosen, hence obtaining 18 projections.

The GRDFS estimate was performed using an order of $17(l=18)$ for which the total calculation time to synthesize the image was $7.12 \mathrm{~s}$. Here lies the main drawback of this method, as it is quite slow when compared to the classical technique which only requires $0.07 \mathrm{~s}$. However, one can clearly see that the synthesized image using the GRDFS approach (Fig. 3(b)) is a lot less noisy than when using the classical approach (Fig. 3(a)). The relative error (err) between synthesized and original images is a good measure for the accuracy of the method. It is given by

$$
\operatorname{err}=\frac{\sum_{x=0}^{N}\left|f_{e s t}(x)-f(x)\right|^{2}}{\sum_{x=0}^{N}|f(x)|^{2}},
$$

where $x$ denotes a running coordinate across each column of the image, $f$ denotes the original image and $f_{\text {est }}$ denotes the synthesized image. The relative error between the true image and the classical approach using truncated interpolation is $16.97 \%$, while using the GRDFS approach it is less at $13.63 \%$. Even when increasing the angular resolution up to 180 projections, the GRDFS approach proves superior with a relative error of $1.87 \%$ while the classical approach shows a relative error of $2.54 \%$ using the truncated interpolation. Using the linear interpolation scheme, the error for the classical technique becomes even larger at $2.90 \%$. In the next section, the approach will be put to an experimental test.

\section{Experimental set-up}

Measurements were performed on a acoustic field originating from a simple loudspeaker $(\emptyset 8 \mathrm{~cm})$, using a Polytec PSV300 SLDV. This measurement principle makes use of the fact that the vibrometer is also sensitive 
a

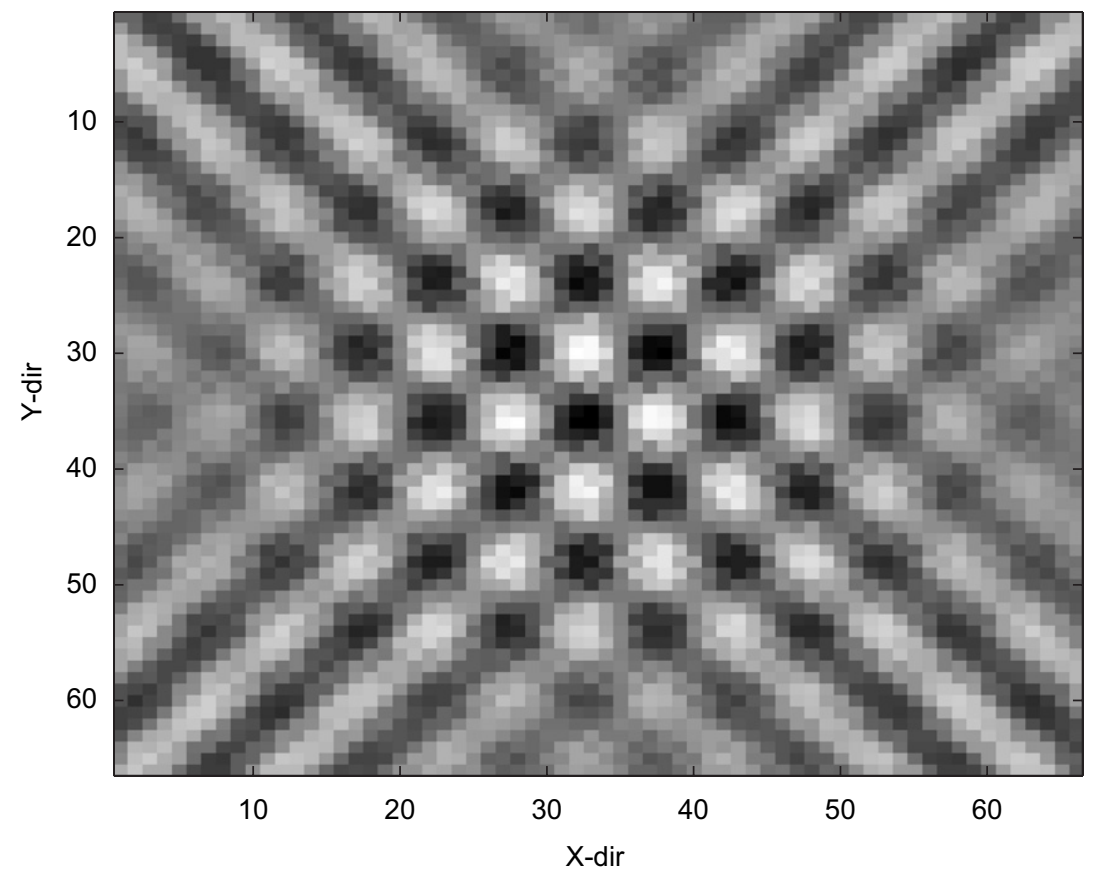

b

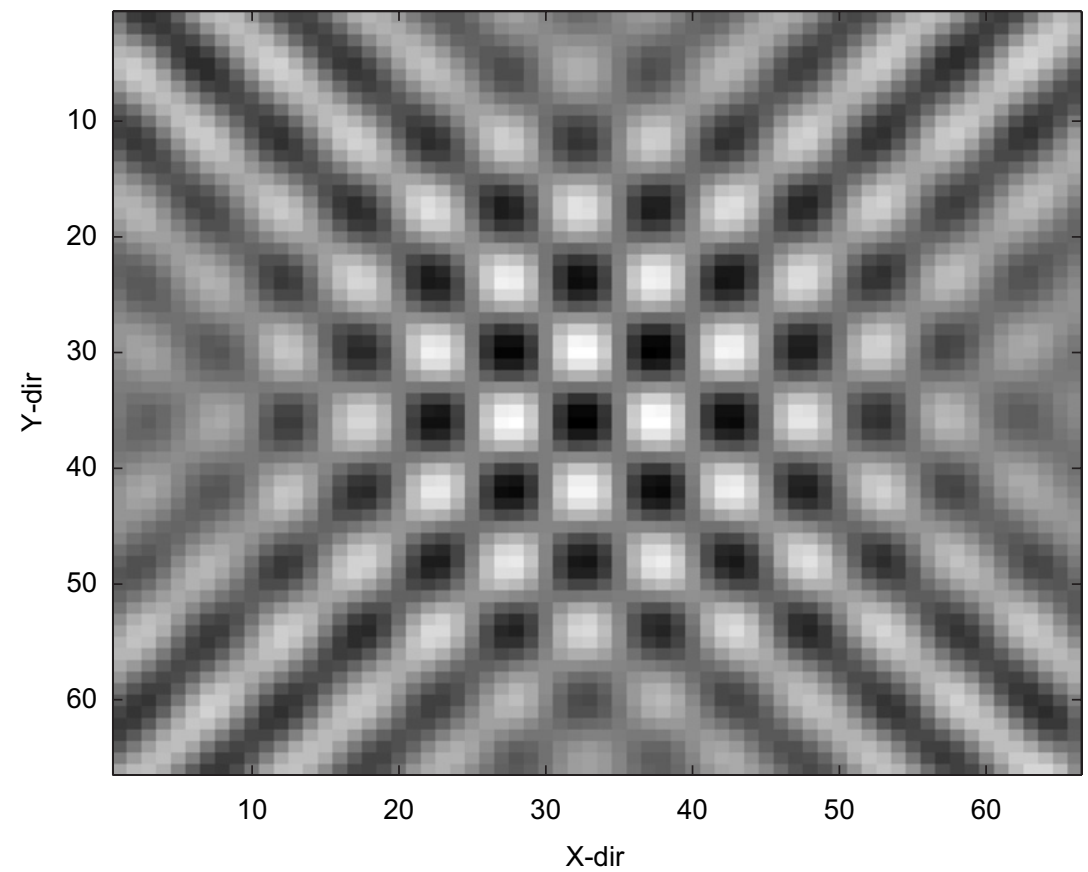

Fig. 3. Synthesized sinusoid from Fig. 2 using 18 projections with: (a) classical filtered back projection with truncated interpolation; (b) GRDFS filtered back projection.

to changes in refractive index of the medium between the laser scanning head and a steady reflective object [6]. By placing a perturbation between the laser head and the rigid object it is possible to visualize that perturbation. Six hundred and fifty scan points were measured on the rigid plate for a single sine excitation 


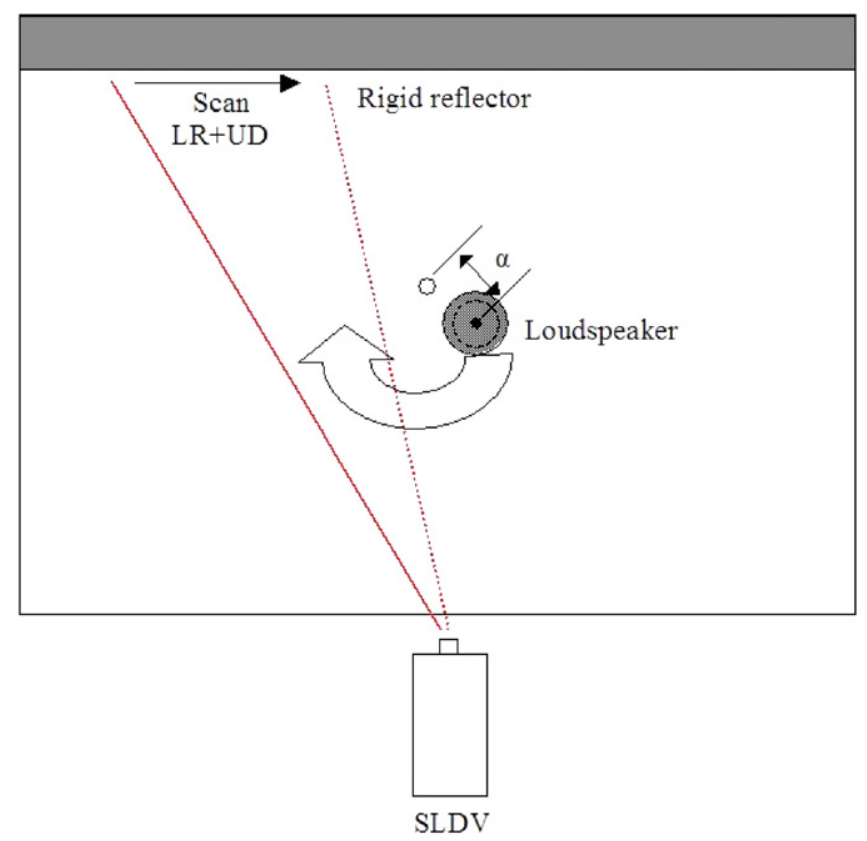

Fig. 4. Schematic view of the test set-up, shown from the top. The SLDV scans left to right (LR) and up and down (UD).

frequency at $10 \mathrm{kHz}$. The rigid plate was $45 \mathrm{~cm}$ wide and $40 \mathrm{~cm}$ high and was covered in retro reflective tape to enhance signal quality. The loudspeaker was placed in the middle between the steady plate and the laser head.

To obtain a number of different angular views, necessary for three-dimensional reconstruction, the acoustic source was placed on a Bruel \& Kjaer type 3923 microphone boom and rotated at its highest velocity of $16 \mathrm{~s} / \mathrm{rev}$. This allows for a continuous (high angular resolution) and automated procedure. For each scan point one entire revolution of the acoustic source is measured in order to have a full $360^{\circ}$ view. The measurement is triggered by means of an Eddy current probe attached to the microphone boom. The rigid reflector was placed on a separate table in order to avoid vibrations caused by the rotating microphone boom. The microphone boom was placed half way between the steady plate and the laser head. Fig. 4 shows a schematic of the measurement set-up. The loudspeaker was placed at two positions, the first $\alpha=0.5 \mathrm{~cm}$ and the second at $\alpha=5 \mathrm{~cm}$ out of axis of rotation.

The obtained signal for each scan point is of course the $10 \mathrm{kHz}$ sine wave but modulated at the rotational frequency of the microphone boom. The acoustic source position is obtained by demodulating this signal.

Because the measured signal is basically an amplitude modulated waveform, it is possible to demodulate this signal electronically. For this purpose a simple peak detector [10] was developed and mounted in between the input and output channel of the vibrometer.

\section{Experimental results}

Two measurements were done with the loudspeaker placed at different positions: 0.5 and $5 \mathrm{~cm}$ away from the axis of the microphone boom. The scan grid contained 625 spatial points $(25 \times 25)$ for both measurements. The grid covered an area on the steady plate of $36 \times 36 \mathrm{~cm}$. Knowing that the microphone boom is exactly in the middle between the LDV and the flat plate, it is easy to determine that the projected measurement grid above the loudspeaker is half the size at $18 \times 18 \mathrm{~cm}$. The measurements were performed with a sample frequency of only $256 \mathrm{~Hz}$, which is possible because of the peak detector, using 2048 spectral lines. This corresponds to a measurement time of exactly $16 \mathrm{~s}$, which is one complete rotational period of the microphone boom. For the next scan point, the following rotation period is discarded and the next period is awaited for a new measurement. 
To reconstruct the full three-dimensional acoustic field the classical filtered back projection method available in the Matlab R12 toolbox together with the GRDFS method was used. Each scan point contains the angle information in the time signal of the amplitudes. This means that for these particular experiments where 2048 DFT lines were used the same number of angles is measured. Combining this angle information from the measured time signal at each scan point together with the spatial information from the scanned grid

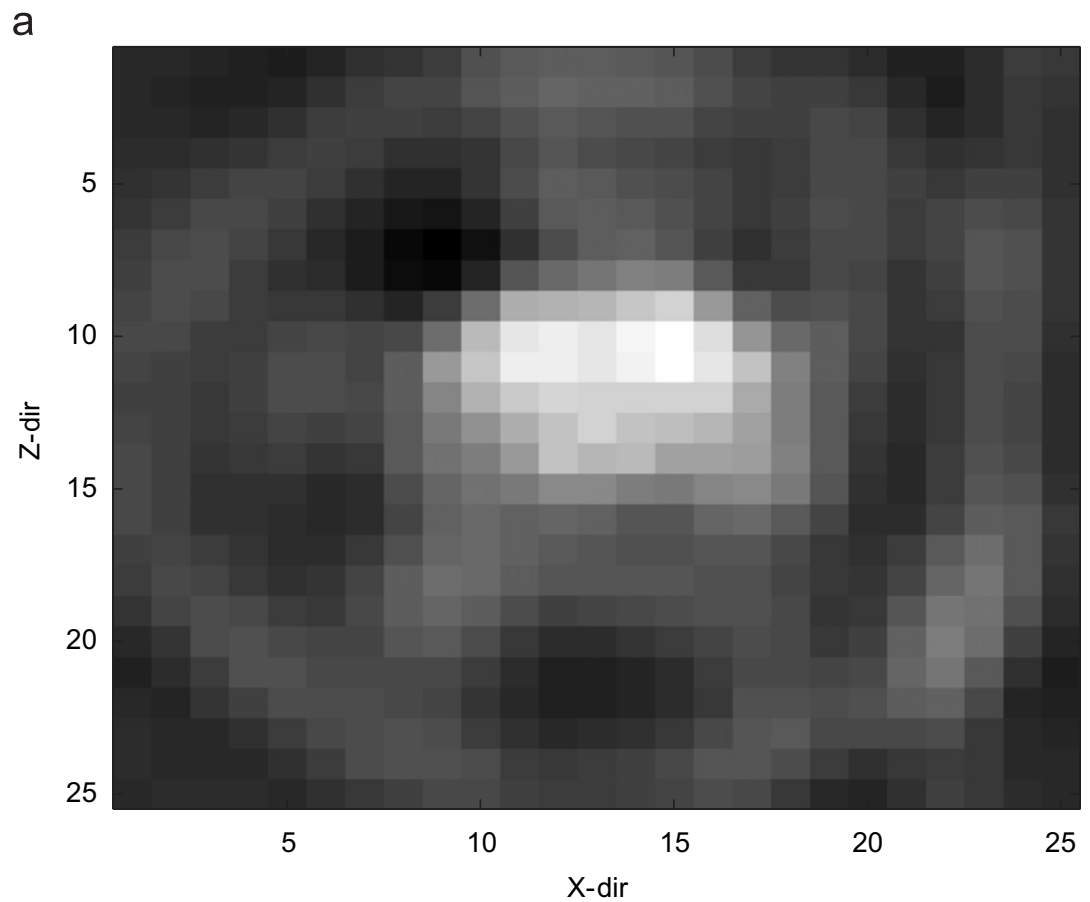

b

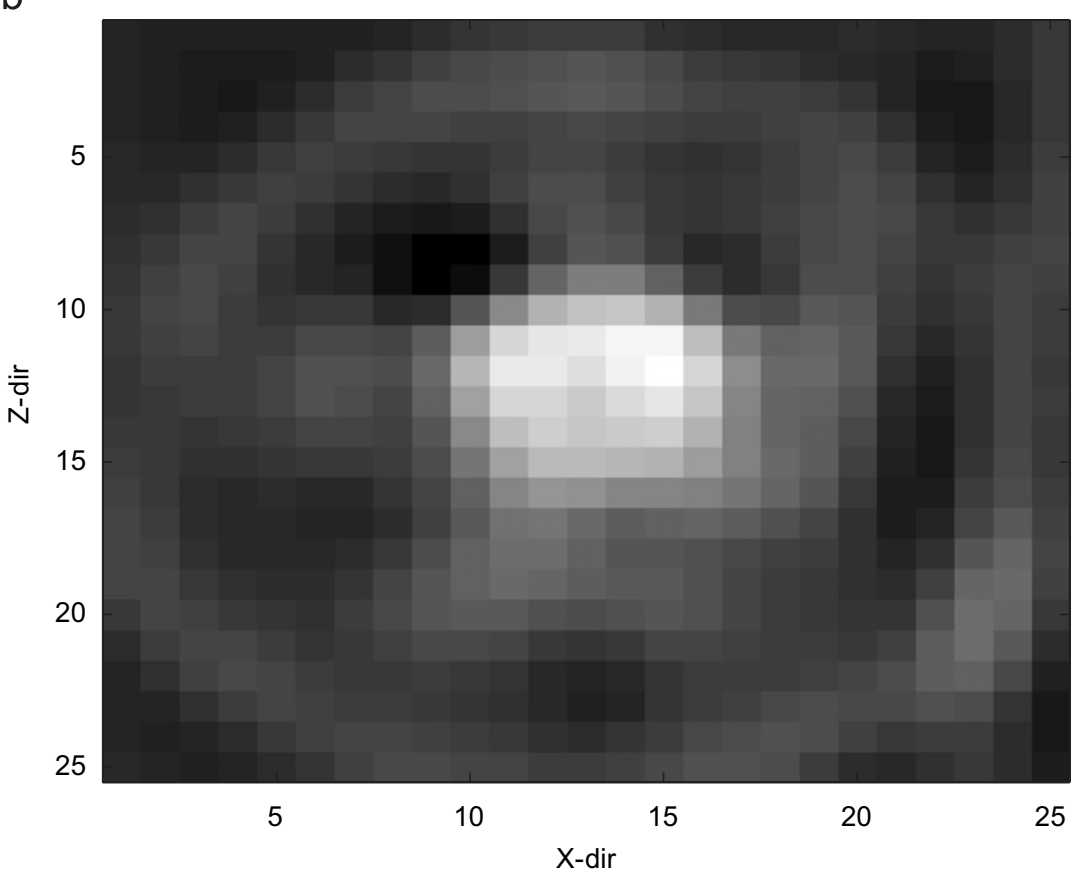

Fig. 5. Acoustic field along a constant $Y$ coordinate $(Y=36 \mathrm{~cm}) ; \alpha=0.5 \mathrm{~cm}$ (a) filtered back projection with linear interpolation (b) GRDFS filtered back projection (axes indicate scan points). 
gives 2048 projections of the acoustic field. This comes down to an angular resolution of $0.176^{\circ}$, which, if done manually, is a tedious job.

In Fig. 5(a), a slice of the field is shown far away from the source $(Y=36 \mathrm{~cm})$ using the classical tomography with the linear interpolation scheme. The bulge near the center indicates the position of the
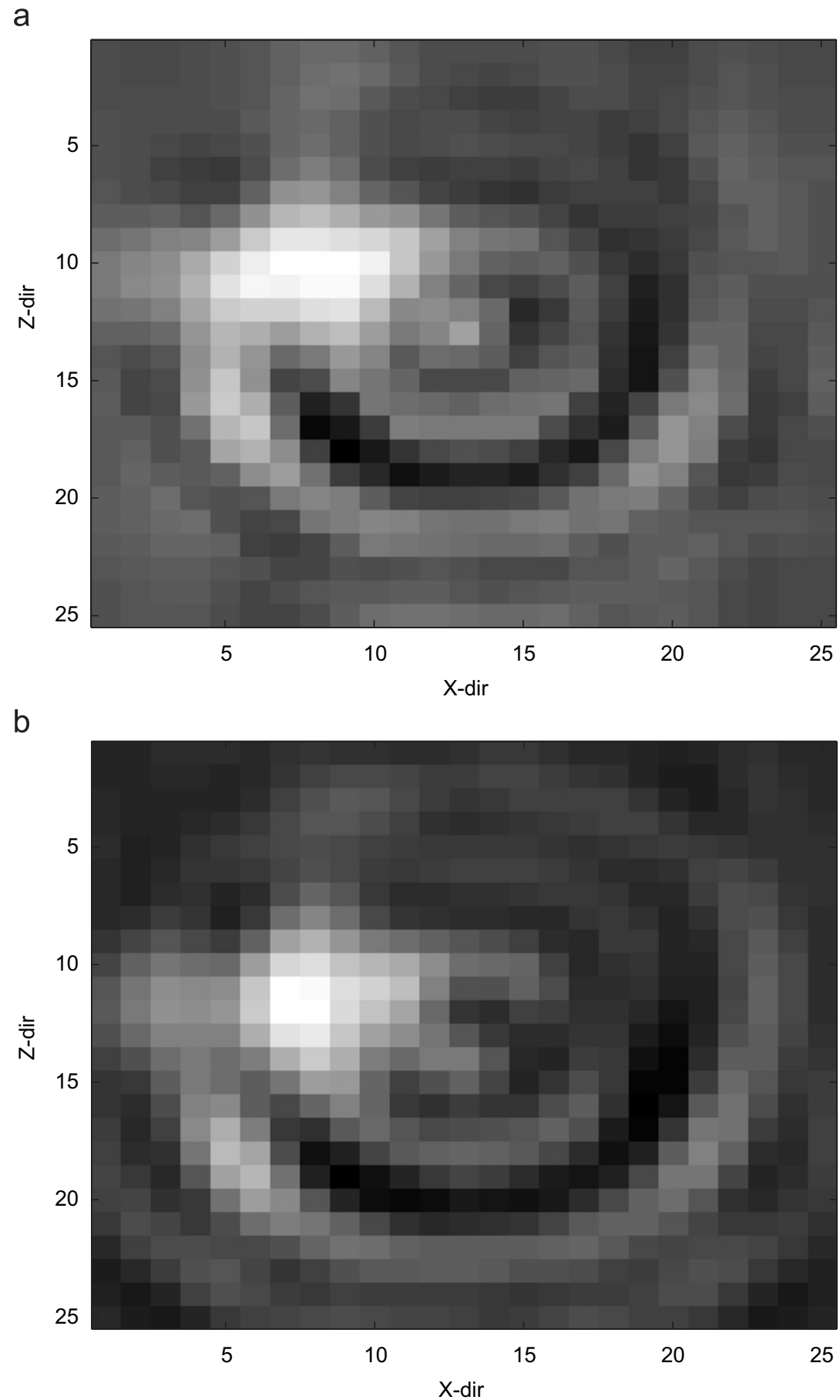

Fig. 6. Acoustic field along a constant $Y$ coordinate $(Y=36 \mathrm{~cm}) ; \alpha=5 \mathrm{~cm}$ (a) filtered back projection with linear interpolation (b) GRDFS filtered back projection (axes indicate scan points). 
loudspeaker. When compared with Fig. 5(b) where the GRDFS scheme is used it is clear that the latter image is less noisy and the bulge is also more compact, making it easier to locate the exact position. This was to be expected as with the traditional scheme, where each back projected image for every angle is an approximation. This leads to blurring in the resulting full image. The pixel position $(x, y)$ of the loudspeaker is determined by means of a weighted average, taking into account the six largest peaks in the synthesize image:

$$
(x, y)=\frac{\sum_{i=1}^{6} a_{i}\left(x_{i}, y_{i}\right)}{\sum_{i=1}^{6} a_{i}},
$$

where $a_{i}$ indicates the amplitude of the $i$ th peak and $\left(x_{i}, y_{i}\right)$ indicate the pixel positions of the six largest peaks. Using simple trigonometry, the distance from the calculated pixel position to the center can be determined.

In case of the classical approach the loudspeaker position can be found to be approximately 1.5 pixels out of center, which comes down to $\alpha=1.08 \mathrm{~cm}$ as compared to the actual $0.5 \mathrm{~cm}$. The GRDFS approach estimates the maximum to be around 1 pixel out of center, which means $\alpha=0.72 \mathrm{~cm}$ again opposed to the actual $0.5 \mathrm{~cm}$.

This previously mentioned blurring is even more pronounced when looking at the second measurement where the acoustic source is placed $5 \mathrm{~cm}$ out of axis (Fig. 6). Using the classical filtered back projection, the estimated location of the acoustic source is a very large area. This makes it quite difficult to pinpoint the source accurately. Using the GRDFS this area is far smaller and the background of the image is also far less noisy. The classical tomography using the linear interpolation scheme estimates the loudspeaker to be 8 pixels or $\alpha=5.76 \mathrm{~cm}$ out of axis while the GRDFS predicts 7 pixels or $\alpha=5.04 \mathrm{~cm}$.

It is clear that the GRDFS performs far better than the classical technique. The only drawback is again the computation time. For one slice of the image, as was shown here, the calculation time for the classical technique is negligible at $0.7 \mathrm{~s}$ on a standard pentium 4 processor, while using the GRDFS it takes $231.24 \mathrm{~s}$. This is of course also due to the very large number of projections.

\section{Conclusions}

In this article a parametric frequency-domain approach, called GRDFS, was applied to improve the accuracy of the standard tomography algorithm. This representation eliminates the necessity of interpolation during back projection in the tomography algorithm, hence improving the accuracy of the synthesis. This novel method was applied to both simulations and laser Doppler vibrometer measurements on a rotating acoustic source. These measurements had a very high angular resolution due to the automated continuous scanning measurement set-up. From these measurements it was proven possible to localize the position of the acoustic source. The proposed method with GRDFS was shown to be more accurate in determining the acoustic location than the existing tomographic algorithm with all its different interpolation schemes. The only drawback of the method proved to be the computation time, due to the iterative nature of the method.

\section{Acknowledgments}

This research has been sponsored by the Fund for Scientific Research-Flanders (FWO) Belgium. The authors also acknowledge the Flemish government (GOA-Optimech) and the research council of the Vrije Universiteit Brussel (OZR) for their funding. J. Vanherzeele holds a grant as postdoctoral researcher from the FWO Flanders.

\section{References}

[1] A. Ramm, A.I. Katsevich, The Radon Transform and Local Tomography, CRC Press, Boca Raton, 1996.

[2] J.R.F. Arruda, Surface smoothing and partial derivatives computation using a regressive discrete Fourier series, Mechanical Systems and Signal Processing 6 (1) (1992) 41-50.

[3] J.R.F. Arruda, S.A.V. Rio, L.A.S.B. Santos, A space-frequency data compression method for spatially dense laser doppler vibrometer measurements, Journal of Shock and Vibration 3 (2) (1996) 127-133.

[4] J. Vanherzeele, P. Guillaume, S. Vanlanduit, Improved Fourier analysis using parametric frequency-domain transfer-function estimators, Mechanical Systems and Signal Processing 21 (4) (2006). 
[5] L. Zipser, H. Franke, Laser-scanning vibrometry for the investigation of sound generation and propagation, in: 6th International Conference on Vibration Measurements by Laser Techniques, SPIE, 2004.

[6] L. Zipser, F.Wachter, H. Franke. Refracto-vibrometry for visualizing sound radiation from vibrating objects, in: P. Sas (ed.), Proceedings of ISMA2006, 2006.

[7] J. Vanherzeele, S. Vanlanduit, P. Guillaume, Acoustic source identification using a scanning ldv, Optics and Lasers in Engineering 45 (6) (2007) 742-749.

[8] P. Guillaume, P. Verboven, S. Vanlanduit, Frequency-domain maximum likelihood identification of modal parameters with confidence intervals, Noise and Vibration Engineering 1 (1998) 359-376.

[9] P. Guillaume, L. Hermans, H.V.D. Auweraer, Maximum likelihood identification of modal parameters from operational data, in: Proceedings of the 17th International Modal Analysis Conference, vol. 2, 1999, pp. 1887-1893.

[10] J. Lesurf, The envelope detector 〈www.st-andrews.ac.uk〉. 